\title{
Global well-posedness for the viscous primitive equations of geophysics
}

Jinyi Sun ${ }^{*}$ and Minghua Yang

${ }^{\text {"Correspondence: }}$ sunjinyi333@163.com Department of Mathematics, Sun Yat-Sen University, Guangzhou, 510275, P.R. China

\begin{abstract}
We study global well-posedness for Cauchy problem of the three-dimensional viscous primitive equations of geophysics in the critical functional framework.

MSC: 35Q35; 35Q86; 76D03

Keywords: viscous primitive equations; global well-posedness; hybrid-Besov spaces
\end{abstract}

\section{Introduction}

The viscous primitive equations are a fundamental mathematical model of geophysics that describes the large-scale ocean and atmosphere dynamics, see, for instance, the monographs [1-3]. The model reads as follows:

$$
\begin{cases}\partial_{t} u-v \Delta u+\Omega e_{3} \times u+(u \cdot \nabla) u+\nabla p=g \theta e_{3} & \text { in } \mathbb{R}^{3} \times(0, \infty) \\ \partial_{t} \theta-\mu \Delta \theta+(u \cdot \nabla) \theta=-\mathcal{N}^{2} u_{3} & \text { in } \mathbb{R}^{3} \times(0, \infty), \\ \operatorname{div} u=0 & \text { in } \mathbb{R}^{3} \times(0, \infty)\end{cases}
$$

where the unknown functions $u=\left(u_{1}, u_{2}, u_{3}\right), p$, and $\theta$ denote the fluid velocity, pressure, and thermal disturbance, respectively, and $v, \mu$, and $g$ are the positive constants of viscosity, thermal diffusivity, and gravity, respectively. Moreover, $\Omega$ is the so-called Coriolis parameter, a real constant which is twice the angular velocity of the rotation around the vertical unit vector $e_{3}=(0,0,1)$, and $\mathcal{N}$ is the stratification parameter, a nonnegative constant representing the Brunt-Väisälä wave frequency. The ratio $P:=\frac{v}{\mu}$ is known as the Prandtl number, and $B:=\frac{\Omega}{N}$ is essentially the "Burger" number of geophysics. We refer the reader to $[1,3,4]$ for derivation of this model and more detailed discussions on its physical background.

If $\theta \equiv 0, \mathcal{N}=0$, and $\Omega=0$, then (1.1) reduces to the classical incompressible NavierStokes equations

$$
\begin{cases}\partial_{t} u-v \Delta u+(u \cdot \nabla) u=-\nabla p & \text { in } \mathbb{R}^{3} \times(0, \infty), \\ \operatorname{div} u=0 & \text { in } \mathbb{R}^{3} \times(0, \infty)\end{cases}
$$

which have drawn great attention during the past fifty more years. It has been proved that the Cauchy problem of (NS) is globally well posed for small initial data in a family of

(c) 2016 Sun and Yang. This article is distributed under the terms of the Creative Commons Attribution 4.0 International License (http://creativecommons.org/licenses/by/4.0/), which permits unrestricted use, distribution, and reproduction in any medium, provided you give appropriate credit to the original author(s) and the source, provide a link to the Creative Commons license, and indicate if changes were made. 
function spaces including particularly the following ones:

$$
\dot{H}^{\frac{1}{2}}\left(\mathbb{R}^{3}\right) \hookrightarrow L^{3}\left(\mathbb{R}^{3}\right) \hookrightarrow \dot{B}_{p, \infty}^{-1+\frac{3}{p}}\left(\mathbb{R}^{3}\right)(3<p<\infty) \hookrightarrow \mathrm{BMO}^{-1}\left(\mathbb{R}^{3}\right) ;
$$

see Fujita and Kato [5], Kato [6], Cannone [7], and Koch and Tataru [8]. These spaces are called critical because their norms are invariant with respect to the following scaling:

$$
\left(u_{\lambda}(t, x), p_{\lambda}(t, x)\right):=\left(\lambda u\left(\lambda^{2} t, \lambda x\right), \lambda^{2} p\left(\lambda^{2} t, \lambda x\right)\right)
$$

which is related to the Navier-Stokes equations themselves. More precisely, if $(u, p)$ is a solution of (NS), so is $\left(u_{\lambda}, p_{\lambda}\right)$. Note that the literatures listed here are far from being complete; we refer the reader to [9] and [10] for exposition and more references. If only $\theta \equiv 0$ and $\mathcal{N}=0$ but $\Omega \neq 0$, then (1.1) reduces to the incompressible rotating Navier-Stokes equations

$$
\begin{cases}\partial_{t} u-v \Delta u+\Omega e_{3} \times u+(u \cdot \nabla) u=-\nabla p & \text { in } \mathbb{R}^{3} \times(0, \infty) \\ \operatorname{div} u=0 & \text { in } \mathbb{R}^{3} \times(0, \infty)\end{cases}
$$

The topic of well-posedness for the Cauchy problem of (RNS) has also been widely studied in various function spaces. We refer the interested reader to [11-21] and the references therein.

In this paper we study the global well-posedness of the Cauchy problem of the viscous primitive equations (1.1), that is, the problem

$$
\begin{cases}\partial_{t} u-v \Delta u+\Omega e_{3} \times u+(u \cdot \nabla) u+\nabla p=g \theta e_{3} & \text { in } \mathbb{R}^{3} \times(0, \infty), \\ \partial_{t} \theta-\mu \Delta \theta+(u \cdot \nabla) \theta=-\mathcal{N}^{2} u_{3} & \text { in } \mathbb{R}^{3} \times(0, \infty), \\ \operatorname{div} u=0 & \text { in } \mathbb{R}^{3} \times(0, \infty), \\ \left.u\right|_{t=0}=u_{0},\left.\quad \theta\right|_{t=0}=\theta_{0} & \text { in } \mathbb{R}^{3} .\end{cases}
$$

Before going further, let us first make a short review on the study of the well-posedness topic of this problem. By taking full advantage of the absence of resonances between the fast rotation and the nonlinear advection, Babin, Mahalov, and Nicolaenko [4] obtained the global well-posedness of problem (1.2) in $H^{s}\left(\mathbb{T}^{3}\right)$ with $s \geq 3 / 4$ for small initial data when the stratification parameter $\mathcal{N}$ is sufficiently large. By constructing the solution of a quasi-geostrophic system related to equations (1.1) and using some Strichartz-type estimates, Charve [22] verified global well-posedness of problem (1.2) in $\dot{H}^{\frac{1}{2}}\left(\mathbb{R}^{3}\right) \cap \dot{H}^{1}\left(\mathbb{R}^{3}\right)$ for arbitrary (i.e., not necessarily small) initial data under the assumptions that both $\Omega$ and $\mathcal{N}$ are sufficiently large (depending on the scale of the initial data). Charve [23] further considered the well-posedness of (1.2) in less regular initial value spaces. We also mention the interesting work of Charve and Ngo [24] on the well-posedness of the problem (1.2) with anisotropic viscosities. Recently, Koba, Mahalov, and Yoneda [25] proved the global wellposedness of problem (1.2) for any given $\left(u_{0}, \theta_{0}\right) \in \dot{H}^{\frac{1}{2}}\left(\mathbb{R}^{3}\right) \cap \dot{H}^{1}\left(\mathbb{R}^{3}\right)$ with $\partial_{2} u_{0}^{1}-\partial_{1} u_{0}^{2}=0$ in the special case where the Prandtl number $P=1$, provided that one of the following conditions holds: (a) $|B|<\sqrt{g}$, and $\mathcal{N}$ is sufficiently large (depending on the scale of the initial data); (b) $|B|>\sqrt{g}$, and both $\Omega$ and $\mathcal{N}$ are sufficiently large (depending on the scale of initial data). They also proved the following global result for uniformly small data with respect to $\Omega$ and $\mathcal{N}$ in $\dot{H}^{\frac{1}{2}}\left(\mathbb{R}^{3}\right)$. 
Theorem 1.1 ([25]) Let $P=1$, that is, $v=\mu$. Then there exists a positive constant $c=c(v)$ such that if $\left(u_{0}, \theta_{0}\right) \in\left[\dot{H}^{\frac{1}{2}}\left(\mathbb{R}^{3}\right)\right]^{4}$ satisfies $\operatorname{div} u_{0}=0$ and

$$
\left\|\left(u_{0}, \theta_{0}\right)\right\|_{\dot{H}^{\frac{1}{2}}} \leq c,
$$

then problem (1.2) has a unique mild solution $(u, \theta) \in\left[C\left([0, \infty) ; \dot{H}^{\frac{1}{2}}\left(\mathbb{R}^{3}\right)\right)\right]^{4} \cap\left[\tilde{L}^{2}(0, \infty\right.$; $\left.\left.\dot{H}^{\frac{3}{2}}\left(\mathbb{R}^{3}\right)\right)\right]^{4}$.

For other related studies on the viscous primitive equations (1.1), we refer the interested reader to [26-31].

For problem (1.2), the situation is obviously more complicated than (NS) and (RNS) on account of the coupling effect between the velocity $u(t, x)$ and the thermal disturbance $\theta(t, x)$. Moreover, due to the influence of the oscillations caused by the rotation (i.e., the term $\Omega e_{3} \times u$ ) and the stratification (i.e., the terms $g \theta e_{3}$ and $\mathcal{N}^{2} u_{3}$ ), a big portion of the integral estimates, such as $L^{p}$ estimate for $p \neq 2$, for the Stokes semigroup $\left\{\mathrm{e}^{t \mathbb{P} \Delta}\right\}_{t \geq 0}$ (which relates to the Navier-Stokes equations) do not work for the Stokes-Coriolis-Stratification semigroup $\left\{T_{\Omega, N}(t)\right\}_{t \geq 0}$ (see Section 2 for the definition) related to the primitive equations. Consequently, the usual function spaces used in the study of the Navier-Stokes equations such as the homogeneous and inhomogeneous Besov spaces $\dot{B}_{p, r}^{s}\left(\mathbb{R}^{3}\right)$ and $B_{p, r}^{s}\left(\mathbb{R}^{3}\right)$ with $p \neq 2$ and the space $\mathrm{BMO}^{-1}\left(\mathbb{R}^{3}\right)$ are not suitable for the primitive equations. In this work, inspired by $[13,32,33]$, we introduce a customized hybrid-Besov space $\dot{B}_{2, p}^{\frac{1}{2}, \frac{3}{p}-1}$, seeing Definition 2.2, in which we shall obtain the regularizing effects of $\left\{T_{\Omega, N}(t)\right\}_{t \geq 0}$ similar to the Stokes semigroup and gain the global solvability for (1.2). Our main result is stated as follows.

Theorem 1.2 Let $P=1$, that is, $v=\mu$, and let $p \in[2,4]$. There exists a positive constant $c$ independent of $\Omega$ and $\mathcal{N}$ such that if $\left(u_{0}, \theta_{0}\right) \in\left[\dot{B}_{2, p}^{\frac{1}{2},-1+\frac{3}{p}}\left(\mathbb{R}^{3}\right)\right]^{4}$ satisfies $\operatorname{div} u_{0}=0$ and

$$
\left\|\left(u_{0}, \sqrt{g} \theta_{0} / \mathcal{N}\right)\right\|_{\dot{B}_{2, p}^{\frac{1}{2},-1+\frac{3}{p}}} \leq c,
$$

then problem (1.2) possesses a unique mild solution $(u, \theta)$ in

$$
\begin{aligned}
& {\left[C\left([0, \infty) ; \dot{B}_{2, p}^{\frac{1}{2},-1+\frac{3}{p}}\left(\mathbb{R}^{3}\right)\right)\right]^{4} \cap\left[\tilde{L}^{\frac{2}{1-\alpha}}\left(0, \infty ; \dot{B}_{2, p}^{\frac{3}{2}-\alpha, \frac{3}{p}-\alpha}\left(\mathbb{R}^{3}\right)\right)\right]^{4}} \\
& \cap\left[\tilde{L}^{\frac{2}{1+\alpha}}\left(0, \infty ; \dot{B}_{2, p}^{\frac{3}{2}+\alpha, \frac{3}{p}+\alpha}\left(\mathbb{R}^{3}\right)\right)\right]^{4}
\end{aligned}
$$

where $\alpha \in\left(\frac{3}{2}-\frac{3}{p}, 1\right]$ is an arbitrary fixed number.

Remark 1.3 Obviously, Theorem 1.2 is an improvement of Theorem 1.1 due to $\dot{H}^{\frac{1}{2}} \hookrightarrow$ $\dot{B}_{2, p}^{\frac{1}{2}, \frac{3}{p}-1}$ for $p \geq 2$. It is also worth mentioning that $\theta_{0}$ can be large in (1.3), provided that $\mathcal{N}$ is large enough.

The rest part of this paper is organized as follows. In Section 2 we introduce the hybridBesov space $\dot{B}_{2, p}^{\frac{1}{2}, \frac{3}{p}-1}$ and Stokes-Coriolis-Stratification semigroup $\left\{T_{\Omega, N}(t)\right\}_{t \geq 0}$ and investigate the regularizing effects of $\left\{T_{\Omega, N}(t)\right\}_{t \geq 0}$. In Section 3, we use the Littlewood-Paley 
analysis technique to derive some linear estimates and a useful product law. Finally, we present the proof of our main result.

Throughout this paper, we use $C$ and $c$ to denote universal constants whose values may change from line to line. Both $\mathcal{F} g$ and $\hat{g}$ stand for the Fourier transform of $g$ with respect to space variable, whereas $\mathcal{F}^{-1}$ stands for the inverse Fourier transform. For any $1 \leq p \leq \infty$, we denote $L^{p}(0, T)$ and $L^{q}\left(\mathbb{R}^{3}\right)$ by $L_{T}^{p}$ and $L^{q}$, respectively.

\section{Function spaces and Stokes-Coriolis-Stratification semigroup}

Let $\delta\left(\mathbb{R}^{3}\right)$ be the Schwartz class, and let $\delta^{\prime}\left(\mathbb{R}^{3}\right)$ be the space of tempered distributions. First, we recall the homogeneous Littlewood-Paley decomposition. Choose two radial functions $\varphi, \psi \in \varsigma\left(\mathbb{R}^{3}\right)$ such that their Fourier transforms $\hat{\varphi}$ and $\hat{\psi}$ satisfy the following properties:

$$
\begin{aligned}
& \operatorname{supp} \hat{\varphi} \subset \mathcal{B}:=\left\{\xi \in \mathbb{R}^{3}:|\xi| \leq \frac{4}{3}\right\}, \\
& \operatorname{supp} \hat{\psi} \subset \mathcal{C}:=\left\{\xi \in \mathbb{R}^{3}: \frac{3}{4} \leq|\xi| \leq \frac{8}{3}\right\},
\end{aligned}
$$

and, furthermore,

$$
\sum_{j \in \mathbb{Z}} \hat{\psi}\left(2^{-j} \xi\right)=1 \quad \text { for all } \xi \in \mathbb{R}^{3} \backslash\{0\}
$$

Let $\varphi_{j}(x):=2^{3 j} \varphi\left(2^{j} x\right)$ and $\psi_{j}(x):=2^{3 j} \psi\left(2^{j} x\right)$ for all $j \in \mathbb{Z}$. We define by $\Delta_{j}$ and $S_{j}$ the following operators in $\varsigma^{\prime}\left(\mathbb{R}^{3}\right)$ :

$$
\Delta_{j} f:=\psi_{j} * f \quad \text { and } \quad S_{j} f:=\varphi_{j} * f \quad \text { for } j \in \mathbb{Z} \text { and } f \in \mathcal{S}^{\prime}\left(\mathbb{R}^{3}\right) .
$$

Define $\delta_{h}^{\prime}\left(\mathbb{R}^{3}\right):=\varsigma^{\prime}\left(\mathbb{R}^{3}\right) / \mathcal{P}\left[\mathbb{R}^{3}\right]$, where $\mathcal{P}\left[\mathbb{R}^{3}\right]$ denotes the linear space of polynomials on $\mathbb{R}^{3}$ (see $[34,35]$ ). It is known that there hold the following decompositions:

$$
f=\sum_{j \in \mathbb{Z}} \Delta_{j} f \quad \text { and } \quad S_{j} f=\sum_{j^{\prime} \leq j-1} \Delta_{j^{\prime}} f \quad \text { in } \delta_{h}^{\prime}\left(\mathbb{R}^{3}\right) .
$$

With our choice of $\varphi$ and $\psi$, it is easy to verify that

$$
\begin{aligned}
& \Delta_{j} \Delta_{k} f=0 \quad \text { if }|j-k| \geq 2 \quad \text { and } \\
& \Delta_{j}\left(S_{k-1} f \Delta_{k} f\right)=0 \quad \text { if }|j-k| \geq 5 .
\end{aligned}
$$

Here, we recall the definition of general homogeneous Besov spaces $\dot{B}_{p, r}^{s}$ and introduce the hybrid-Besov space $\dot{B}_{2, p}^{\sigma, \beta}$ and the Chemin-Lerner-type spaces $\tilde{L}_{T}^{\delta}\left(0, \infty ; \dot{B}_{2, p}^{\sigma, \beta}\left(\mathbb{R}^{3}\right)\right)$, which are made to measure problem (1.2).

Definition 2.1 ([35]) Let $s \in \mathbb{R}, 1 \leq p, r \leq \infty$, and $u \in \wp_{h}^{\prime}\left(\mathbb{R}^{3}\right)$. We set

$$
\|u\|_{\dot{B}_{p, r}^{s}}:=\left\|\left\{2^{j s}\left\|\Delta_{j} u\right\|_{L^{p}}\right\}_{r \in \mathbb{Z}}\right\|_{\ell^{r}(\mathbb{Z})} .
$$


- For $s<\frac{3}{p}$ (or $s=\frac{3}{p}$ if $r=1$ ), we define $\dot{B}_{p, r}^{s}\left(\mathbb{R}^{3}\right):=\left\{u \in \mathcal{S}_{h}^{\prime}\left(\mathbb{R}^{3}\right):\|u\|_{\dot{B}_{p, r}^{s}}<\infty\right\}$;

- If $k \in \mathbb{N}$ and $\frac{3}{p}+k \leq s<\frac{3}{p}+k+1$ (or $s=\frac{3}{p}+k+1$ if $r=1$ ), then $\dot{B}_{p, r}^{s}\left(\mathbb{R}^{3}\right)$ is defined as the subset of distributions $u \in \mathcal{S}_{h}^{\prime}\left(\mathbb{R}^{3}\right)$ such that $\partial^{\delta} u \in \dot{B}_{p, r}^{s-k}\left(\mathbb{R}^{3}\right)$ whenever $|\delta|=k$.

Definition 2.2 Let $N:=\mathcal{N} \sqrt{g}, \sigma, \beta \in \mathbb{R}$, and $1 \leq p \leq \infty$. Then the hybrid-Besov space $\dot{B}_{2, p}^{\sigma, \beta}$ is defined by

$$
\dot{B}_{2, p}^{\sigma, \beta}\left(\mathbb{R}^{3}\right):=\left\{u \in \wp_{h}^{\prime}\left(\mathbb{R}^{3}\right):\|u\|_{\dot{B}_{2, p}^{\sigma, \beta}}<\infty\right\},
$$

where

$$
\|u\|_{\dot{B}_{2, p}^{\sigma, \beta}}:=\sup _{2^{j} \leq \max \{|\Omega|, N\}} 2^{j \sigma}\left\|\Delta_{j} f\right\|_{L^{2}}+\sup _{2^{j}>\max \{|\Omega|, N\}} 2^{j \beta}\left\|\Delta_{j} f\right\|_{L^{p}} .
$$

Definition 2.3 Let $N:=\mathcal{N} \sqrt{g}$. For $\sigma, \beta \in \mathbb{R}$ and $1 \leq p, \delta \leq \infty$, we set

$$
\|u\|_{\tilde{L}_{T}^{\delta}\left(\dot{B}_{2, p}^{\sigma, \beta}\right)}:=\sup _{2^{j} \leq \max \{|\Omega|, N\}} 2^{j \sigma}\left\|\Delta_{j} f\right\|_{L_{T}^{\delta} L^{2}}+\sup _{2^{j}>\max \{|\Omega|, N\}} 2^{j \beta}\left\|\Delta_{j} f\right\|_{L_{T}^{\delta} L^{p}} .
$$

We then define the space $\tilde{L}^{\delta}\left(0, T ; \dot{B}_{2, p}^{\sigma, \beta}\left(\mathbb{R}^{3}\right)\right)$ as the set of temperate distributions $u$ over $(0, T) \times \mathbb{R}^{3}$ such that $\lim _{j \rightarrow-\infty} S_{j} u=0$ in $\delta^{\prime}\left((0, T) \times \mathbb{R}^{3}\right)$ and $\|u\|_{\tilde{L}_{T}^{\delta}\left(\dot{B}_{2, p}^{\sigma, \beta}\right)}<\infty$.

In the sequel, we will constantly use the following Bernstein inequality.

Lemma 2.4 ([34,35]) Let $\mathcal{B}$ be a ball, and $\mathcal{C}$ a ring centered at origin in $\mathbb{R}^{3}$. There exists a constant $C$ such that for any positive real number $\lambda$, any nonnegative integer $k$, and any couple of real numbers $(a, b)$ with $b \geq a \geq 1$, we have:

- Supp $\hat{u} \subset \lambda \mathcal{B} \Longrightarrow \sup _{|\alpha|=k}\left\|\partial^{\alpha} u\right\|_{L^{b}} \leq C^{k+1} \lambda^{k+3\left(\frac{1}{a}-\frac{1}{b}\right)}\|u\|_{L^{a}}$;

- Supp $\hat{u} \subset \lambda \mathcal{C} \Longrightarrow C^{-(k+1)} \lambda^{-k}\|u\|_{L^{a}} \leq \sup _{|\alpha|=k}\left\|\partial^{\alpha} u\right\|_{L^{a}} \leq C^{k+1} \lambda^{k}\|u\|_{L^{a}}$.

Now, we introduce the Stokes-Coriolis-Stratification semigroup $\left\{T_{\Omega, N}(t)\right\}_{t \geq 0}$ and study its regularizing effects.

By setting $N:=\mathcal{N} \sqrt{g}, v:=\left(v^{1}, v^{2}, v^{3}, v^{4}\right):=\left(u^{1}, u^{2}, u^{3}, \sqrt{g} \theta / \mathcal{N}\right), v_{0}:=\left(v_{0}^{1}, v_{0}^{2}, v_{0}^{3}, v_{0}^{4}\right):=$ $\left(u_{0}^{1}, u_{0}^{2}, u_{0}^{3}, \sqrt{g} \theta_{0} / \mathcal{N}\right)$, and $\widetilde{\nabla}:=\left(\partial_{1}, \partial_{2}, \partial_{3}, 0\right)$ problem (1.2) can be rewritten as the following problem:

$$
\begin{cases}\partial_{t} v+\mathcal{A} v+\mathcal{B} v+\widetilde{\nabla} p=-(v \cdot \widetilde{\nabla}) v & \text { in } \mathbb{R}^{3} \times(0, \infty), \\ \widetilde{\nabla} \cdot v=0 & \text { in } \mathbb{R}^{3} \times(0, \infty), \\ \left.v\right|_{t=0}=v_{0} & \text { in } \mathbb{R}^{3},\end{cases}
$$

where

$$
\mathcal{A}:=\left(\begin{array}{cccc}
-\nu \Delta & 0 & 0 & 0 \\
0 & -\nu \Delta & 0 & 0 \\
0 & 0 & -\nu \Delta & 0 \\
0 & 0 & 0 & -\mu \Delta
\end{array}\right), \quad \mathcal{B}:=\left(\begin{array}{cccc}
0 & -\Omega & 0 & 0 \\
\Omega & 0 & 0 & 0 \\
0 & 0 & 0 & -N \\
0 & 0 & N & 0
\end{array}\right) .
$$

Lemma 3.3 in [25], together with the fact $e^{(\mathcal{A}+\mathcal{B}) t}=e^{\mathcal{A} t} e^{\mathcal{B} t}$ for $v=\mu$, gives an explicit expression of the Stokes-Coriolis-Stratification semigroup $\left\{T_{\Omega, N}(t)\right\}_{t \geq 0}$ corresponding to the 
linear problem of (2.1) via the Fourier transform

$$
\begin{aligned}
T_{\Omega, N}(t) f:= & \mathcal{F}^{-1}\left[\cos \left(\frac{|\xi|^{\prime}}{|\xi|} t\right) e^{-\nu|\xi|^{2} t} M_{1}(\xi) \hat{f}+\sin \left(\frac{|\xi|^{\prime}}{|\xi|} t\right) e^{-\nu|\xi|^{2} t} M_{2}(\xi) \hat{f}\right. \\
& \left.+e^{-v|\xi|^{2} t} M_{3}(\xi) \hat{f}\right]
\end{aligned}
$$

where

$$
|\xi|:=\sqrt{\xi_{1}^{2}+\xi_{2}^{2}+\xi_{3}^{2}}, \quad|\xi|^{\prime}:=|\xi|_{\Omega, N}^{\prime}:=\sqrt{N^{2} \xi_{1}^{2}+N^{2} \xi_{2}^{2}+\Omega^{2} \xi_{3}^{2}}
$$

for $\xi:=\left(\xi_{1}, \xi_{2}, \xi_{3}\right) \in \mathbb{R}^{3}$, and

$$
\begin{aligned}
& M_{1}(\xi):=\left(\begin{array}{cccc}
\frac{\Omega^{2} \xi_{3}^{2}}{|\xi|^{\prime 2}} & 0 & -\frac{N^{2} \xi_{1} \xi_{3}}{|\xi|^{\prime 2}} & \frac{\Omega N \xi_{2} \xi_{3}}{|\xi|^{2}} \\
0 & \frac{\Omega^{2} \xi_{3}^{2}}{|\xi|^{2}} & -\frac{N^{2} \xi_{2} \xi_{3}}{|\xi|^{\prime 2}} & -\frac{\Omega N \xi_{1} \xi_{3}}{|\xi|^{2}} \\
-\frac{\Omega^{2} \xi_{1} \xi_{3}}{|\xi|^{2}} & -\frac{\Omega^{2} \xi_{2} \xi_{3}}{|\xi|^{\prime 2}} & \frac{N^{2}\left(\xi_{1}^{2}+\xi_{2}^{2}\right)}{|\xi|^{2}} & 0 \\
\frac{\Omega N \xi_{2} \xi_{3}}{|\xi|^{\prime 2}} & -\frac{\Omega N \xi_{1} \xi_{3}}{|\xi|^{\prime 2}} & 0 & \frac{N^{2}\left(\xi_{1}^{2}+\xi_{2}^{2}\right)}{|\xi|^{2}}
\end{array}\right), \\
& M_{2}(\xi):=\left(\begin{array}{cccc}
0 & -\frac{\Omega \xi_{3}^{2}}{\left.|\xi| \xi\right|^{\prime}} & \frac{\Omega \xi_{2} \xi_{3}}{\left.|\xi| \xi\right|^{\prime}} & \frac{N \xi_{1} \xi_{3}}{\left.|\xi| \xi\right|^{\prime}} \\
\frac{\Omega \xi_{3}^{2}}{\left.|\xi| \xi\right|^{\prime}} & 0 & -\frac{\Omega \xi_{1} \xi_{3}}{|\xi \| \xi|^{\prime}} & \frac{N \xi_{2} \xi_{3}}{|\xi \| \xi|^{\prime}} \\
-\frac{\Omega \xi_{2} \xi_{3}}{\left.|\xi| \xi\right|^{\prime}} & \frac{\Omega \xi_{1} \xi_{3}}{|\xi||\xi|^{\prime}} & 0 & -\frac{N\left(\xi_{1}^{2}+\xi_{2}^{2}\right)}{\left.|\xi| \xi\right|^{\prime}} \\
-\frac{N \xi_{1} \xi_{3}}{|\xi||\xi|^{\prime}} & -\frac{N \xi_{2} \xi_{3}}{\left.|\xi| \xi\right|^{\prime}} & \frac{N\left(\xi_{1}^{2}+\xi_{2}^{2}\right)}{\left.|\xi| \xi\right|^{\prime}} & 0
\end{array}\right),
\end{aligned}
$$

and

$$
M_{3}(\xi):=\left(\begin{array}{cccc}
\frac{N^{2} \xi_{2}^{2}}{|\xi|^{\prime 2}} & -\frac{N^{2} \xi_{1} \xi_{2}}{|\xi|^{\prime 2}} & 0 & -\frac{N \Omega \xi_{2} \xi_{3}}{|\xi|^{\prime 2}} \\
-\frac{N^{2} \xi_{1} \xi_{2}}{|\xi|^{2}} & \frac{N^{2} \xi_{1}^{2}}{|\xi|^{2}} & 0 & \frac{N \Omega \xi_{1} \xi_{3}}{|\xi|^{2}} \\
0 & 0 & 0 & 0 \\
-\frac{N \Omega \xi_{2} \xi_{3}}{|\xi|^{2}} & \frac{N \Omega \xi_{1} \xi_{3}}{|\xi|^{2}} & 0 & \frac{\Omega^{2} \xi_{3}^{2}}{|\xi|^{2}}
\end{array}\right) .
$$

Note that, denoting by $M_{j k}^{l}(\xi)(j, k=1,2,3,4, l=1,2,3)$ the $(j, k)$ th component of the matrix $M_{l}(\xi)$, it is obvious that nonvanishing $M_{j k}^{l}(\xi)$ satisfies

$$
\left|M_{j k}^{l}(\xi)\right| \leq 2 \quad \text { for } \xi \in \mathbb{R}^{3}, j, k=1,2,3,4, l=1,2,3
$$

Hence, from (2.3) and Plancherel's theorem it is easy to see that $\left\{T_{\Omega, N}(t)\right\}_{t \geq 0}$ is a bounded $C_{0}$-semigroup on $L^{2}\left(\mathbb{R}^{3}\right)$. By Mikhlin's theorem we may extend the semigroup $\left\{T_{\Omega, N}(t)\right\}_{t \geq 0}$ to a $C_{0}$-semigroup on $L^{p}\left(\mathbb{R}^{3}\right)$ for $1<p<\infty$. Moreover, we have

$$
\left\|T_{\Omega, N}(t) f\right\|_{L^{p}} \leq C_{p} \max \{|\Omega|, N\}^{2} t^{2}\|f\|_{L^{p}}, \quad t \geq 1, f \in L^{p}\left(\mathbb{R}^{3}\right)
$$

for some constant $C_{p}$. However, it is noteworthy that $T_{\Omega, N}$ is not uniformly bounded in $L^{p}\left(\mathbb{R}^{3}\right)$ for $p \neq 2$, which is the primary reason for the invalidation of Cannone's proof [7] in $\dot{B}_{p, r}^{s}\left(\mathbb{R}^{3}\right)$ with $p \neq 2$ for our case, and similarly for $B_{p, r}^{s}\left(\mathbb{R}^{3}\right)$ and $\mathrm{BMO}^{-1}\left(\mathbb{R}^{3}\right)$. 
Thanks to the Euler formula $e^{ \pm i \frac{|\xi|^{\prime}}{\xi \xi} t}=\cos \left(\frac{|\xi|^{\prime}}{|\xi|} t\right) \pm i \sin \left(\frac{|\xi|^{\prime}}{|\xi|} t\right)$, we can rewrite the semigroup $\left\{T_{\Omega, N}(t)\right\}_{t \geq 0}$ as

$$
T_{\Omega, N}(t) f:=\frac{1}{2} e^{i \frac{|D|^{\prime}}{|D|} t} e^{\nu t \Delta}\left(\mathcal{M}_{1}+\mathcal{M}_{2}\right) f+\frac{1}{2} e^{-i \frac{|D|^{\prime}}{|D|} t} e^{\nu t \Delta}\left(\mathcal{M}_{1}-\mathcal{M}_{2}\right) f+e^{\nu t \Delta} \mathcal{M}_{3} f
$$

where $\frac{|D|^{\prime}}{|D|}$ is the Fourier multiplier with symbol given by $\frac{|\xi|^{\prime}}{|\xi|}$, and $\mathcal{M}_{i}(i=1,2,3)$ with symbols given by $M_{i}(\xi)(i=1,2,3)$ are the matrices of singular integral operators. The operators $e^{ \pm i \frac{|D|^{\prime}}{|D|^{\prime}} t}$ represent the oscillation parts of $\left\{T_{\Omega, N}(t)\right\}_{t \geq 0}$.

By considering low and high frequencies differently, we can establish the following smoothing effect of the Stokes-Coriolis-Stratification semigroup $\left\{T_{\Omega, N}(t)\right\}_{t \geq 0}$.

Lemma 2.5 Let $\mathcal{C}$ be a annulus centered at 0 in $\mathbb{R}^{3}$. Then there exist positive constants $c$ and $C$ depending only on $v$ such that if $\operatorname{supp} \hat{u} \subset \lambda \mathcal{C}$, then we have

(i) for any $\lambda>0$,

$$
\left\|T_{\Omega, N}(t) u\right\|_{L^{2}} \leq e^{-c \lambda^{2} t}\|u\|_{L^{2}}
$$

(ii) for any $\lambda \gtrsim \max \{|\Omega|, N\}$ and $1 \leq p \leq \infty$,

$$
\left\|T_{\Omega, N}(t) u\right\|_{L^{p}} \leq e^{-c \lambda^{2} t}\|u\|_{L^{p}}
$$

Proof (i) By Plancherel's theorem, combining the support property of $\hat{u}$, it is obvious that (2.9) is obtained directly from expression (2.3).

(ii) Decomposing $T_{\Omega, N}(t)$ into $T_{\Omega, N}(t):=T_{\Omega, N}^{1}(t)+T_{\Omega, N}^{2}(t)$, where

$$
T_{\Omega, N}^{1}(t) f:=\frac{1}{2} e^{i \frac{|D|^{\prime}}{|D|} t} e^{\nu t \Delta}\left(\mathcal{M}_{1}+\mathcal{M}_{2}\right) f+\frac{1}{2} e^{-i \frac{|D|^{\prime}}{|D|} t} e^{\nu t \Delta}\left(\mathcal{M}_{1}-\mathcal{M}_{2}\right) f
$$

and

$$
T_{\Omega, N}^{2}(t) f:=e^{v t \Delta} \mathcal{M}_{3} f
$$

For $T_{\Omega, N}^{2}(t)$, since each nonvanishing component of $M_{3}(\xi)$ is homogeneous with degree 0 , Fourier multiplier theory implies that $\mathcal{M}_{3}$ is bounded in $L^{p}(1 \leq p \leq \infty)$ when localized in dyadic annulus in the Fourier space. Applying Lemma 2.4 in [34] yields

$$
\left\|T_{\Omega, N}^{2}(t) u\right\|_{L^{p}} \leq e^{-c \lambda^{2} t}\|u\|_{L^{p}}
$$

Now, we focus our attention on $T_{\Omega, N}^{1}(t)$. We will adopt the spirit of the proof for the heat operator as in [34]. Let $\phi \in D\left(\mathbb{R}^{3} \backslash\{0\}\right)$ be equal to 1 near the annulus $\mathcal{C}$. Set

$$
T(t, x):=\mathcal{F}^{-1}\left[\phi\left(\lambda^{-1} \xi\right) \hat{T}_{\Omega, N}^{1}(t, \xi)\right](t, x)=(2 \pi)^{-3} \int_{\mathbb{R}^{3}} e^{i x \cdot \xi} \phi\left(\lambda^{-1} \xi\right) \hat{T}_{\Omega, N}^{1}(t, \xi) d \xi .
$$

Thus, to prove (2.10), it suffices to show that

$$
\|T(t, \cdot)\|_{L^{1}} \leq C e^{-c \lambda^{2} t}
$$


Thanks to the boundedness properties of $M_{1}(\xi)$ and $M_{2}(\xi)$, we have

$$
\int_{|x| \leq \lambda^{-1}}|T(t, x)| d x \leq C \int_{|x| \leq \lambda^{-1}} \int_{\mathbb{R}^{3}}\left|\phi\left(\lambda^{-1} \xi\right)\right|\left|\hat{T}_{\Omega, N}^{1}(t, \xi)\right| d \xi d x \leq C e^{-c \lambda^{2} t} .
$$

Let $L:=\frac{x \cdot \nabla_{\xi}}{i|x|^{2}}$. It is easy to check that $L\left(e^{i x \cdot \xi}\right)=e^{i x \cdot \xi}$. By integration by parts we obtain

$$
\begin{aligned}
T(t, x) & =(2 \pi)^{-3} \int_{\mathbb{R}^{3}} L^{m}\left(e^{i x \cdot \xi}\right) \phi\left(\lambda^{-1} \xi\right) \hat{T}_{\Omega, N}^{1}(t, \xi) d \xi \\
& =(2 \pi)^{-3} \int_{\mathbb{R}^{3}} e^{i x \cdot \xi}\left(L^{*}\right)^{m}\left(\phi\left(\lambda^{-1} \xi\right) \hat{T}_{\Omega, N}^{1}(t, \xi)\right) d \xi,
\end{aligned}
$$

where $m \in \mathbb{N}$ is chosen later. We verify by applying the Leibnitz formula that

$$
\left|\partial^{\gamma}\left(e^{ \pm i|\xi|^{\prime}} t\right)\right| \leq C|\xi|^{-\gamma}(\max \{|\Omega|, N\} t+1)^{|\gamma|}
$$

and

$$
\left|\partial^{\gamma}\left(e^{-v|\xi|^{2} t}\right)\right| \leq C|\xi|^{-\gamma} e^{-\frac{v}{2}|\xi|^{2} t}
$$

Thus, we have

$$
\begin{aligned}
& \left|\left(L^{*}\right)^{m}\left(\phi\left(\lambda^{-1} \xi\right) \hat{T}_{\Omega, N}^{1}(t, \xi)\right)\right| \\
& \leq C|x|^{-m} \sum_{\substack{\left|\alpha_{1}\right|+\left|\alpha_{2}\right|+\left|\alpha_{3}\right|=|\alpha| \\
|\alpha| \leq m}} \lambda^{-(m-|\alpha|)} \mid\left(\nabla^{m-|\alpha|} \phi\right)\left(\lambda^{-1} \xi\right) \partial^{\alpha_{1}}\left(e^{ \pm i|\xi|^{\prime}} t\right) \\
& \quad \times \partial^{\alpha_{2}}\left(e^{-\nu|\xi|^{2} t}\right) \partial^{\alpha_{3}}\left(M_{1}(\xi)+M_{2}(\xi)\right) \mid \\
& \leq C|\lambda x|^{-m} \sum_{\substack{\left|\alpha_{1}\right|+\left|\alpha_{2}\right|+\left|\alpha_{3}\right|=|\alpha| \\
|\alpha| \leq m}} \lambda^{|\alpha|}\left|\left(\nabla^{m-|\alpha|} \phi\right)\left(\lambda^{-1} \xi\right)\right||\xi|^{-\left|\alpha_{1}\right|-\left|\alpha_{2}\right|-\left|\alpha_{3}\right|} e^{-\frac{v}{4}|\xi|^{2} t} \\
& \quad \times(\max \{|\Omega|, N\} t+1)^{\left|\alpha_{1}\right|} .
\end{aligned}
$$

Taking $m=4$ for $\lambda \gtrsim\{|\Omega|, N\}$ in

$$
\left|\left(L^{*}\right)^{m}\left(\phi\left(\lambda^{-1} \xi\right) \hat{T}_{\Omega, N}^{1}(t, \xi)\right)\right| \leq C|\lambda x|^{-4} e^{-\frac{v}{4}|\xi|^{2} t}
$$

leads to

$$
\int_{|\lambda| \geq \frac{1}{\lambda}}|T(t, x)| d x \leq C e^{-c \lambda^{2} t} \lambda^{3} \int_{|\lambda| \geq \frac{1}{\lambda}}|\lambda x|^{-4} d x \leq C e^{-c \lambda^{2} t}
$$

which, together with (2.12), gives (2.11). Inequality (2.10) is proved.

\section{Linear estimates and bilinear estimates}

We establish some basic estimates that will play a crucial role in the proof of Theorem1.2. We first consider linear estimates for the semigroup $\left\{T_{\Omega, N}(t)\right\}_{t \geq 0}$. 
Lemma 3.1 Let $T>0, \alpha \in[0,1], \sigma, \beta \in \mathbb{R}$, and $p \in[1, \infty]$. Then, for $u \in \dot{B}_{2, p}^{\sigma, \beta}\left(\mathbb{R}^{3}\right)$, there exists a constant $C>0$ such that

$$
\left\|T_{\Omega, N}(t) u\right\|_{\tilde{L}^{\frac{2}{1 \pm \alpha}}\left(0, T ; \dot{B}_{2, p}^{\sigma+1 \pm \alpha, \beta+1 \pm \alpha}\right)} \leq C\|u\|_{\dot{B}_{2, p}^{\sigma, \beta}}
$$

Proof For $j$ such that $2^{j}>\max \{|\Omega|, N\}$, by Lemma 2.5 we have

$$
\|\| \Delta_{j} T_{\Omega, N}(t) u\left\|_{L^{p}}\right\|_{L^{1 \pm \alpha}(0, T)} \leq C\left\|e^{-c 2^{2 j} t}\right\| \Delta_{j} u\left\|_{L^{p}}\right\|_{L^{1 \pm \alpha}(0, T)}^{2} \leq C 2^{-(1 \pm \alpha) j}\left\|\Delta_{j} u\right\|_{L^{p}}
$$

Similarly, for $j$ such that $2^{j} \leq \max \{|\Omega|, N\}$, we have

$$
\|\| \Delta_{j} T_{\Omega, N}(t) u\left\|_{L^{2}}\right\|_{L^{1 \pm \alpha}(0, T)} \leq C 2^{-(1 \pm \alpha) j}\left\|\Delta_{j} u\right\|_{L^{2}}
$$

Combining (3.2) with (3.3) yields (3.1).

Lemma 3.2 Let $T>0, \alpha \in[0,1], \sigma, \beta \in \mathbb{R}$, and $p \in[1, \infty]$. There exists a constant $C>0$ such that

$$
\left\|\int_{0}^{t} T_{\Omega, N}(t-\tau) f(\tau) d \tau\right\|_{\tilde{L}^{\frac{2}{1 \pm \alpha}\left(0, T ; \dot{B}_{2, p}^{\sigma+1 \pm \alpha, \beta+1 \pm \alpha}\right)}} \leq C\|f\|_{\tilde{L}^{1}\left(0, T ; \dot{B}_{2, p}^{\sigma, \beta}\right)}
$$

for any $f \in \tilde{L}^{1}\left(0, T ; \dot{B}_{2, p}^{\sigma, \beta}\left(\mathbb{R}^{3}\right)\right)$.

Proof For $j$ such that $2^{j}>\max \{|\Omega|, N\}$, applying Lemma 2.5 and Young's inequality yields

$$
\begin{aligned}
\left\|\Delta_{j} \int_{0}^{t} T_{\Omega, N}(t-\tau) f(\tau) d \tau\right\|_{L^{1 \pm \alpha}\left(0, T ; L^{p}\right)} & \leq C\left\|\int_{0}^{t} e^{-c 2^{2 j}(t-\tau)}\right\| \Delta_{j} f(\tau)\left\|_{L^{p}} d \tau\right\|_{L^{1 \pm \alpha}} \\
& \leq C 2^{-(1 \pm \alpha) j}\left\|\Delta_{j} f\right\|_{L^{1}\left(0, T ; L^{p}\right)} .
\end{aligned}
$$

Similarly, for $j$ such that $2^{j} \leq \max \{|\Omega|, N\}$, we obtain

$$
\left\|\Delta_{j} \int_{0}^{t} T_{\Omega, N}(t-\tau) f(\tau) d \tau\right\|_{L^{\frac{2}{1 \pm \alpha}\left(0, T ; L^{2}\right)}} \leq C 2^{-(1 \pm \alpha) j}\left\|\Delta_{j} f\right\|_{L^{1}\left(0, T ; L^{2}\right)} .
$$

Inequality (3.5), together with (3.6), yields (3.4).

We now turn to establish the following product law, which is indispensable for gaining the bilinear estimate in the proof of our main result.

Lemma 3.3 Let $T>0, p \in[2,4]$, and $\alpha \in\left(\frac{3}{2}-\frac{3}{p}, 1\right]$. There exists a constant $C>0$ such that

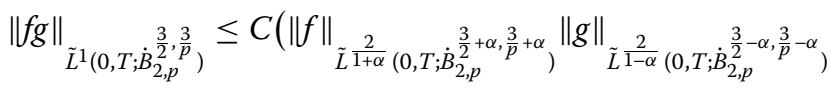

$$
\begin{aligned}
& \left.\left.+\|g\|_{\tilde{L}^{1+\alpha}\left(0, T ; \dot{B}_{2, p}^{2}+\alpha, \frac{3}{p}+\alpha\right.}\right)\|f\|_{\tilde{L}^{1-\alpha}\left(0, T ; \dot{B}_{2, p}^{2}-\alpha, \frac{3}{p}-\alpha\right.}\right)
\end{aligned}
$$

for all $f, g \in \tilde{L}^{\frac{2}{ \pm \alpha}}\left(0, T ; \dot{B}_{2, p}^{\frac{3}{2} \pm \alpha, \frac{3}{p} \pm \alpha}\left(\mathbb{R}^{3}\right)\right)$. 
Proof Applying Bony's decomposition [36], we rewrite $\dot{\Delta}_{j}(f g)$ as

$$
\begin{aligned}
\dot{\Delta}_{j}(f g) & =\sum_{|k-j| \leq 4} \dot{\Delta}_{j}\left(\dot{S}_{k-1} f \dot{\Delta}_{k} g\right)+\sum_{|k-j| \leq 4} \dot{\Delta}_{j}\left(\dot{S}_{k-1} f \dot{\Delta}_{k} g\right)+\sum_{k \geq j-2} \sum_{\left|k-k^{\prime}\right| \leq 1} \dot{\Delta}_{j}\left(\dot{\Delta}_{k} f \dot{\Delta}_{k^{\prime}} g\right) \\
& =: I_{j}+I I_{j}+I I I_{j} .
\end{aligned}
$$

First, we consider $I_{j}$. Set $K_{j}:=\left\{\left(k^{\prime}, k\right) ;|k-j| \leq 4, k^{\prime} \leq k-2\right\}$. On the one hand, for $2^{j}>$ $\max \{|\Omega|, N\}$, we have

$$
\begin{aligned}
\left\|I_{j}\right\|_{L_{T}^{1} L^{p}} & \leq \sum_{K_{j}}\left\|\Delta_{j}\left(\Delta_{k^{\prime}} f \Delta_{k} g\right)\right\|_{L_{T}^{1} L^{p}} \\
& \leq\left(\sum_{K_{j, l l}}+\sum_{K_{j, l h}}+\sum_{K_{j, h h}}\right)\left\|\Delta_{j}\left(\Delta_{k^{\prime}} f \Delta_{k} g\right)\right\|_{L_{T^{1}}^{1} L^{p}} \\
& =: I_{j, 1}+I_{j, 2}+I_{j, 3}
\end{aligned}
$$

where

$$
\begin{aligned}
& K_{j, l l}:=\left\{\left(k^{\prime}, k\right) \in K_{j} ; 2^{k^{\prime}} \leq \max \{|\Omega|, N\}, 2^{k} \leq \max \{|\Omega|, N\}\right\}, \\
& K_{j, l h}:=\left\{\left(k^{\prime}, k\right) \in K_{j} ; 2^{k^{\prime}} \leq \max \{|\Omega|, N\}, 2^{k}>\max \{|\Omega|, N\}\right\}, \\
& K_{j, h h}:=\left\{\left(k^{\prime}, k\right) \in K_{j} ; 2^{k^{\prime}}>\max \{|\Omega|, N\}, 2^{k}>\max \{|\Omega|, N\}\right\} .
\end{aligned}
$$

Applying Lemma 2.4 and Hölder's inequality, we see that

$$
\begin{aligned}
& I_{j, 1} \leq \sum_{\left(k^{\prime}, k\right) \in K_{j, l} l}\left\|\Delta_{j}\left(\Delta_{k^{\prime}} f \Delta_{k} g\right)\right\|_{L_{T}^{1} L^{p}} \\
& \leq \sum_{\left(k^{\prime}, k\right) \in K_{j, l l}} 2^{\frac{3}{2} k^{\prime}}\left\|\Delta_{k^{\prime}} f\right\|_{L_{T}^{\frac{2}{1-\alpha}} L^{2}} 2^{k\left(\frac{3}{2}-\frac{3}{p}\right)}\left\|\Delta_{k} g\right\|_{L_{T}^{1+\alpha} L^{2}} \\
& \leq \sum_{\left(k^{\prime}, k\right) \in K_{j, l l}} 2^{k^{\prime}\left(\frac{3}{2}-\alpha\right)}\left\|\Delta_{k^{\prime}} f\right\|_{L_{T}^{1-\alpha} L^{2}} 2^{k\left(\frac{3}{2}+\alpha\right)}\left\|\Delta_{k} g\right\|_{L_{T}^{1+\alpha} L^{2}} 2^{\alpha\left(k^{\prime}-k\right)} 2^{-\frac{3}{p} k} \\
& \leq C\|f\|_{L_{T}^{1-\alpha} \dot{B}_{2, p}^{2}-\alpha, \frac{3}{p}-\alpha}\|g\|_{L_{T}^{1+\alpha}}^{\frac{2}{B_{2, p}^{2}}} \dot{3}^{\frac{3}{2}+\alpha, \frac{3}{p}+\alpha} \sum_{\left(k^{\prime}, k\right) \in K_{j, l l}} 2^{\alpha\left(k^{\prime}-k\right)} 2^{-\frac{3}{p} k} \\
& \leq C 2^{-\frac{3}{p} j}\|f\|_{L_{T}^{1-\alpha} \dot{B}_{2, p}^{2}} \frac{3}{2-\alpha, \frac{3}{p}-\alpha}\|g\|_{L_{T}^{1+\alpha}} \frac{2}{\dot{B}_{2, p}^{2}+\alpha, \frac{3}{p}+\alpha},
\end{aligned}
$$

where we have used the fact that

$$
\sum_{\left(k^{\prime}, k\right) \in K_{j, l l}} 2^{\alpha\left(k^{\prime}-k\right)} 2^{-\frac{3}{p} k} \leq \sum_{k^{\prime} \leq k-2} 2^{\alpha\left(k^{\prime}-k\right)} \sum_{|k-j| \leq 4} 2^{-\frac{3}{p} k} \leq C 2^{-\frac{3}{p} j} .
$$

Similarly, we have

$$
\begin{aligned}
I_{j, 2} & \leq \sum_{\left(k^{\prime}, k\right) \in K_{j, l h}}\left\|\Delta_{k^{\prime}} f\right\|_{L_{T}^{\frac{1}{1-\alpha}} L^{\infty}}\left\|\Delta_{k} g\right\|_{L_{T}^{\frac{1+\alpha}{1+\alpha}} L^{p}} \\
& \leq C \sum_{\left(k^{\prime}, k\right) \in K_{j, l h}} 2^{\frac{3}{2} k^{\prime}}\left\|\Delta_{k^{\prime}} f\right\|_{L_{T}^{1-\alpha} L^{2}}\left\|\Delta_{k} g\right\|_{L_{T}^{1+\alpha} L^{p}}
\end{aligned}
$$




$$
\begin{aligned}
& =C \sum_{\left(k^{\prime}, k\right) \in K_{j, l h}} 2^{k^{\prime}\left(\frac{3}{2}-\alpha\right)}\left\|\Delta_{k^{\prime}} f\right\|_{L_{T}^{\frac{2}{1-\alpha}} L^{2}} 2^{k\left(\frac{3}{p}+\alpha\right)}\left\|\Delta_{k} g\right\|_{L_{T}^{\frac{2}{1+\alpha}} L^{p}} 2^{\alpha\left(k^{\prime}-k\right)} 2^{-\frac{3}{p} k} \\
& \leq C 2^{-\frac{3}{p} j}\|f\|_{L_{T}^{1-\alpha} \dot{B}_{2, p}^{2}}^{\frac{3}{2}-\alpha, \frac{3}{p}-\alpha}\|g\|_{L_{T}^{1+\alpha} \dot{B}_{2, p}^{2+\alpha, \frac{3}{p}+\alpha}}
\end{aligned}
$$

and

$$
\begin{aligned}
& I_{j, 3} \leq \sum_{\left(k^{\prime}, k\right) \in K_{j, h h}}\left\|\Delta_{k^{\prime}} f\right\|_{L_{T}^{\frac{1}{1-\alpha}} L^{\infty}}\left\|\Delta_{k} g\right\|_{L_{T}^{\frac{1}{1+\alpha}} L^{p}} \\
& \leq C \sum_{\left(k^{\prime}, k\right) \in K_{j, h h}} 2^{\frac{3}{p} k^{\prime}}\left\|\Delta_{k^{\prime}} f\right\|_{L_{T}^{\frac{1}{1-\alpha}} L^{p}}\left\|\Delta_{k} g\right\|_{L_{T}^{1+\alpha} L^{p}} \\
& =C \sum_{\left(k^{\prime}, k\right) \in K_{j, h h}} 2^{k^{\prime}\left(\frac{3}{p}-\alpha\right)}\left\|\Delta_{k^{\prime}} f\right\|_{L_{T}^{1-\alpha} L^{p}} 2^{k\left(\frac{3}{p}+\alpha\right)}\left\|\Delta_{k} g\right\|_{L_{T}^{1+\alpha} L^{p}} 2^{\alpha\left(k^{\prime}-k\right)} 2^{-\frac{3}{p} k} \\
& \leq C 2^{-\frac{3}{p} j}\|f\|_{L_{T}^{1-\alpha} \dot{B}_{2, p}^{2}-\alpha, \frac{3}{p}-\alpha}\|g\|_{L_{T}^{1+\alpha} \dot{B}_{2, p}^{\frac{3}{2}+\alpha, \frac{3}{p}+\alpha}} .
\end{aligned}
$$

On the other hand, for $j$ such that $2^{j} \leq \max \{|\Omega|, N\}$, we have

$$
\begin{aligned}
\left\|I_{j}\right\|_{L_{T}^{1} L^{2}} & \leq \sum_{K_{j}}\left\|\Delta_{j}\left(\Delta_{k^{\prime}} f \Delta_{k} g\right)\right\|_{L_{T}^{1} L^{2}} \\
& \leq\left(\sum_{K_{j, l l}}+\sum_{K_{j, l h}}+\sum_{K_{j, h h}}\right)\left\|\Delta_{j}\left(\Delta_{k^{\prime}} f \Delta_{k} g\right)\right\|_{L_{T^{1}}^{1} L^{2}} \\
& =: I_{j, 4}+I_{j, 5}+I_{j, 6} .
\end{aligned}
$$

Applying Lemma 2.4 and Hölder's inequality gives

$$
\begin{aligned}
& I_{j, 4} \leq \sum_{\left(k^{\prime}, k\right) \in K_{j, l l}}\left\|\Delta_{j}\left(\Delta_{k^{\prime}} f \Delta_{k} g\right)\right\|_{L_{T}^{1} L^{2}} \\
& \leq \sum_{\left(k^{\prime}, k\right) \in K_{j, l l}} 2^{\frac{3}{2} k^{\prime}}\left\|\Delta_{k^{\prime}} f\right\|_{L_{T}^{\frac{1}{1-\alpha}} L^{2}}\left\|\Delta_{k} g\right\|_{L_{T}^{1+\alpha} L^{2}} \\
& \leq \sum_{\left(k^{\prime}, k\right) \in K_{j, l l}} 2^{k^{\prime}\left(\frac{3}{2}-\alpha\right)}\left\|\Delta_{k^{\prime}} f\right\|_{L_{T}^{1-\alpha} L^{2}} 2^{k\left(\frac{3}{2}+\alpha\right)}\left\|\Delta_{k} g\right\|_{L_{T}^{1+\alpha} L^{2}} 2^{\alpha\left(k^{\prime}-k\right)} 2^{-\frac{3}{2} k} \\
& \leq C\|f\|_{L_{T}^{1-\alpha} \dot{B}_{2, p}^{2}-\alpha, \frac{3}{p}-\alpha}\|g\|{ }_{L_{T}^{1+\alpha} \dot{B}_{2, p}^{\frac{3}{2}+\alpha, \frac{3}{p}+\alpha}} \sum_{\left(k^{\prime}, k\right) \in K_{j, l l}} 2^{\alpha\left(k^{\prime}-k\right)} 2^{-\frac{3}{2} k} \\
& \leq C 2^{-\frac{3}{2} j}\|f\|_{L_{T}^{1-\alpha} \dot{B}_{2, p}^{2}-\alpha, \frac{3}{p}-\alpha}\|g\|_{L_{T}^{1+\alpha} \dot{B}_{2, p}^{2}+\alpha, \frac{3}{p}+\alpha},
\end{aligned}
$$

and due to $p \geq 2$ and $\alpha+\frac{3}{p}-\frac{3}{2}>0$, we see that

$$
\begin{aligned}
I_{j, 5} & \leq \sum_{\left(k^{\prime}, k\right) \in K_{j, l h}}\left\|\Delta_{k^{\prime}} f\right\|_{L_{T}^{1-\alpha}} \frac{2 p}{L^{p-2}}\left\|\Delta_{k} g\right\|_{L_{T}^{1+\alpha} L^{p}} \\
& \leq C \sum_{\left(k^{\prime}, k\right) \in K_{j, l h}} 2^{\frac{3}{p^{1}} k^{\prime}}\left\|\Delta_{k^{\prime}} f\right\|_{L_{T}^{1-\alpha} L^{2}}\left\|\Delta_{k} g\right\|_{L_{T}^{1+\alpha} L^{p}}
\end{aligned}
$$




$$
\begin{aligned}
& =C \sum_{\left(k^{\prime}, k\right) \in K_{j, l h}} 2^{k^{\prime}\left(\frac{3}{2}-\alpha\right)}\left\|\Delta_{k^{\prime}} f\right\|_{L_{T}^{\frac{1}{1-\alpha}} L^{2}} 2^{k\left(\frac{3}{p}+\alpha\right)}\left\|\Delta_{k} g\right\|_{L_{T}^{\frac{1}{1+\alpha}} L^{p}} 2^{\left(\alpha+\frac{3}{p}-\frac{3}{2}\right)\left(k^{\prime}-k\right)} 2^{-\frac{3}{2} k} \\
& \leq C 2^{-\frac{3}{2} j}\|f\|_{L_{T}^{\frac{2}{1-\alpha}} \dot{B}_{2, p}^{2}} \frac{3}{2-\alpha, \frac{3}{p}-\alpha}\|g\|_{L_{T}^{\frac{2}{1+\alpha}} \dot{B}_{2, p}^{\frac{3}{2}+\alpha, \frac{3}{p}+\alpha},}
\end{aligned}
$$

and due to $p \leq 4$ and $\alpha+\frac{3}{p}-\frac{3}{2}>0$, we get

$$
\begin{aligned}
I_{j, 6} & \leq \sum_{\left(k^{\prime}, k\right) \in K_{j, h h}}\left\|\Delta_{k^{\prime}} f\right\|_{L_{T}^{\frac{1}{1-\alpha}} \frac{2}{L^{p-2}}}\left\|\Delta_{k} g\right\|_{L_{T}^{\frac{1}{1+\alpha}} L^{p}} \\
& \leq C \sum_{\left(k^{\prime}, k\right) \in K_{j, h h}} 2^{\left(-\frac{3}{2}+\frac{6}{p}\right) k^{\prime}}\left\|\Delta_{k^{\prime}} f\right\|_{L_{T}^{\frac{1}{1-\alpha}} L^{p}}\left\|\Delta_{k} g\right\|_{L_{T}^{\frac{1}{1+\alpha}} L^{p}} \\
& =C \sum_{\left(k^{\prime}, k\right) \in K_{j, h h}} 2^{k^{\prime}\left(\frac{3}{p}-\alpha\right)}\left\|\Delta_{k^{\prime}} f\right\|_{L_{T}^{1-\alpha} L^{p}} 2^{k\left(\frac{3}{p}+\alpha\right)}\left\|\Delta_{k} g\right\|_{L_{T}^{1+\alpha} L^{p}} 2^{\left(\alpha+\frac{3}{p}-\frac{3}{2}\right)\left(k^{\prime}-k\right)} 2^{-\frac{3}{2} k} \\
& \leq C 2^{-\frac{3}{2} j}\|f\|_{L_{T}^{\frac{1}{1-\alpha}} \dot{B}_{2, p}^{\frac{3}{2}-\alpha, \frac{3}{p}-\alpha}}\|g\|_{L_{T}^{\frac{1}{1+\alpha}} \dot{B}_{2, p}^{\frac{3}{2}+\alpha, \frac{3}{p}+\alpha}} .
\end{aligned}
$$

Summing the estimates obtained, $I_{j, 1} \sim I_{j, 6}$ yields that

$$
\begin{aligned}
& \sup _{2^{j}>\max \{|\Omega|, N\}} 2^{\frac{3}{p} j}\left\|I_{j}\right\|_{\tilde{L}_{T}^{1} L^{p}}+\sup _{2^{j} \leq \max \{|\Omega|, N\}} 2^{\frac{3}{2} j}\left\|I_{j}\right\|_{\tilde{L}_{T}^{1} L^{2}} \\
& \leq C\|f\|_{L_{T}^{\frac{1}{1-\alpha}} \dot{B}_{2, p}^{\frac{3}{2}-\alpha, \frac{3}{p}-\alpha}}\|g\|_{L_{T}^{1+\alpha} \dot{B}_{2, p}^{\frac{3}{2}+\alpha, \frac{3}{p}+\alpha}} .
\end{aligned}
$$

By the same argument we have

$$
\begin{aligned}
& \sup _{2^{j}>\max \{|| \Omega \mid, N\}} 2^{\frac{3}{p} j}\left\|I I_{j}\right\|_{\tilde{L}_{T}^{1} L^{p}}+\sup _{2^{j} \leq \max \{|\Omega|, N\}} 2^{\frac{3}{2} j}\left\|I I_{j}\right\|_{\tilde{L}_{T}^{1} L^{2}} \\
& \quad \leq C\|g\| \frac{2}{L_{T}^{1-\alpha} \dot{B}_{2, p}^{\frac{3}{2}-\alpha, \frac{3}{p}-\alpha}}\|f\|_{L_{T}^{1+\alpha} \dot{B}_{2, p}^{\frac{3}{2}+\alpha, \frac{3}{p}+\alpha}} .
\end{aligned}
$$

Now, we consider $I I I_{j}$. Setting $\tilde{K}_{j}:=\left\{\left(k^{\prime}, k\right) ; k \geq j-2,\left|k-k^{\prime}\right| \leq 1\right\}$, we get

$$
\begin{aligned}
I I I_{j} & =\left(\sum_{\tilde{K}_{j, l l}}+\sum_{\tilde{K}_{j, l h}}+\sum_{\tilde{K}_{j, h l}}+\sum_{\tilde{K}_{j, h h}}\right) \Delta_{j}\left(\Delta_{k} f \Delta_{k^{\prime}} g\right) \\
& =: I I I_{j, 1}+I I I_{j, 2}+I I I_{j, 3}+I I I_{j, 4},
\end{aligned}
$$

where

$$
\begin{gathered}
\tilde{K}_{j, l l}:=\left\{\left(k^{\prime}, k\right) \in \tilde{K}_{j} ; 2^{k^{\prime}} \leq \max \{|\Omega|, N\}, 2^{k} \leq \max \{|\Omega|, N\}\right\}, \\
\tilde{K}_{j, l h}:=\left\{\left(k^{\prime}, k\right) \in \tilde{K}_{j} ; 2^{k^{\prime}} \leq \max \{|\Omega|, N\}, 2^{k}>\max \{|\Omega|, N\}\right\}, \\
\tilde{K}_{j, h l}:=\left\{\left(k^{\prime}, k\right) \in \tilde{K}_{j} ; 2^{k^{\prime}}>\max \{|\Omega|, N\}, 2^{k} \leq \max \{|\Omega|, N\}\right\}, \\
\tilde{K}_{j, h h}:=\left\{\left(k^{\prime}, k\right) \in \tilde{K}_{j} ; 2^{k^{\prime}}>\max \{|\Omega|, N\}, 2^{k}>\max \{|\Omega|, N\}\right\} .
\end{gathered}
$$


By Lemma 2.4 and Hölder's inequality we have

$$
\begin{aligned}
& \left\|I I I_{j, 1}\right\|_{L_{T}^{1} L^{p}} \\
& \leq C 2^{3 j\left(1-\frac{1}{p}\right)} \sum_{\left(k^{\prime}, k\right) \in \tilde{K}_{j, l l}}\left\|\Delta_{k} f \Delta_{k^{\prime}} g\right\|_{L_{T}^{1} L^{1}} \\
& \leq C 2^{3 j\left(1-\frac{1}{p}\right)} \sum_{\left(k^{\prime}, k\right) \in \tilde{K}_{j, l l}} 2^{\left(\frac{3}{2}-\alpha\right) k}\left\|\Delta_{k} f\right\| \sum_{L_{T}^{1-\alpha} L^{2}} 2^{\left(\frac{3}{2}+\alpha\right) k^{\prime}}\left\|\Delta_{k^{\prime}} g\right\| \underbrace{}_{L_{T}^{1+\alpha}} L^{2} 2^{-\left(\frac{3}{2}-\alpha\right) k} 2^{-\left(\frac{3}{2}+\alpha\right) k^{\prime}} \\
& \leq C 2^{3 j\left(1-\frac{1}{p}\right)}\|f\|_{L_{T}^{1-\alpha} \dot{B}_{2, p}^{\frac{3}{2}-\alpha, \frac{3}{p}-\alpha}\|g\|} \frac{2}{L_{T}^{1+\alpha}} \dot{B}_{2, p}^{\frac{3}{2}+\alpha, \frac{3}{p}+\alpha} \sum_{k \geq j-3} 2^{-\left(\frac{3}{2}-\alpha\right) k} \sum_{\left|k-k^{\prime}\right| \leq 1} 2^{-\left(\frac{3}{2}+\alpha\right) k^{\prime}} \\
& \leq C 2^{-\frac{3}{p} j}\|f\| \frac{2}{L_{T}^{1-\alpha}} \dot{B}_{2, p}^{\frac{3}{2}-\alpha, \frac{3}{p}-\alpha}\|g\| \frac{2}{L_{T}^{1+\alpha}} \dot{B}_{2, p}^{\frac{3}{2}+\alpha, \frac{3}{p}+\alpha}
\end{aligned}
$$

and

$$
\left\|I I I_{j, 1}\right\|_{L_{T}^{1} L^{2}} \leq C 2^{\frac{3}{2} j} \sum_{\left(k^{\prime}, k\right) \in \tilde{K}_{j, l l}}\left\|\Delta_{k} f \Delta_{k^{\prime}} g\right\|_{L_{T}^{1} L^{1}} \leq C 2^{-\frac{3}{2} j}\|f\|_{L_{T}^{1-\alpha} \dot{B}_{2, p}^{\frac{3}{2}-\alpha, \frac{3}{p}-\alpha}}\|g\|_{L_{T}^{\frac{1}{1+\alpha}} \dot{B}_{2, p}^{\frac{3}{2}+\alpha, \frac{3}{p}+\alpha}}
$$

Similarly, we have

$$
\begin{aligned}
& \left\|I I I_{j, 2}+I I I_{j, 3}\right\|_{L_{T^{L}}^{1} L^{p}} \\
& \leq C 2^{\frac{3}{2} j}\left(\sum_{\left(k^{\prime}, k\right) \in \tilde{K}_{j, l h}}+\sum_{\left(k^{\prime}, k\right) \in \tilde{K}_{j, h l}}\right)\left\|\Delta_{k} f \Delta_{k^{\prime}} g\right\|_{L_{T}^{1} L^{\frac{2 p}{2+p}}} \\
& \leq C 2^{\frac{3}{2} j}\left\{\sum_{\left(k^{\prime}, k\right) \in \tilde{K}_{j, l h}}\left\|\Delta_{k} f\right\|_{L_{T}^{1-\alpha} L^{p}}\left\|\Delta_{k^{\prime}} g\right\|_{L_{T}^{\frac{1}{1+\alpha}} L^{2}}+\sum_{\left(k^{\prime}, k\right) \in \tilde{K}_{j, h l}}\left\|\Delta_{k} f\right\|_{L_{T}^{\frac{1}{1-\alpha}} L^{2}}\left\|\Delta_{k^{\prime}} g\right\|_{L_{T}^{1+\alpha}} L^{p}\right\} \\
& \leq C 2^{\frac{3}{2} j}\|f\| \sum_{L_{T}^{\frac{2}{1-\alpha}} \dot{B}_{2, p}^{\frac{3}{2}-\alpha, \frac{3}{p}-\alpha}\|g\|} \| \frac{2}{L_{T}^{1+\alpha}} \dot{B}_{2, p}^{\frac{3}{2}+\alpha, \frac{3}{p}+\alpha} \\
& \quad \times \sum_{k \geq j-3} \sum_{\left|k-k^{\prime}\right| \leq 1}\left(2^{-\left(\frac{3}{p}-\alpha\right) k} 2^{-\left(\frac{3}{2}+\alpha\right) k^{\prime}}+2^{-\left(\frac{3}{2}+\alpha\right) k} 2^{-\left(\frac{3}{p}-\alpha\right) k^{\prime}}\right) \\
& \leq C 2^{-\frac{3}{p} j}\|f\|{ }_{L_{T}^{\frac{2}{1-\alpha}}} \dot{B}_{2, p}^{\frac{3}{2}-\alpha, \frac{3}{p}-\alpha}\|g\|_{L_{T}^{1+\alpha}} \dot{B}_{2, p}^{\frac{3}{2}+\alpha, \frac{3}{p}+\alpha}
\end{aligned}
$$

and

$$
\begin{aligned}
\left\|I I I_{j, 2}+I I I_{j, 3}\right\|_{L_{T}^{1} L^{2}} & \leq C 2^{\frac{3}{p} j} \sum_{\left(k^{\prime}, k\right) \in \tilde{K}_{j, l l}}\left\|\Delta_{k} f \Delta_{k^{\prime}} g\right\|_{L_{T}^{1} L^{1}} \\
& \leq C 2^{-\frac{3}{2} j}\|f\|_{L_{T}^{\frac{2}{1-\alpha}} \dot{B}_{2, p}^{\frac{3}{2}-\alpha, \frac{3}{p}-\alpha}\|g\|_{L_{T}^{\frac{1}{1+\alpha}} \dot{B}_{2, p}^{\frac{3}{2}+\alpha, \frac{3}{p}+\alpha}}} .
\end{aligned}
$$

Finally, noticing that $4 \geq p \geq 2$, we obtain

$$
\begin{aligned}
& \left\|I I I_{j, 4}\right\|_{L_{T}^{1} L^{p}} \\
& \leq C 2^{\frac{3}{p} j} \sum_{\left(k^{\prime}, k\right) \in \tilde{K}_{j, h h}}\left\|\Delta_{k} f \Delta_{k^{\prime}} g\right\|_{L_{T^{1}}^{1} L^{\frac{p}{2}}}
\end{aligned}
$$




$$
\begin{aligned}
& \leq C 2^{\frac{3}{p} j} \sum_{\left(k^{\prime}, k\right) \in \tilde{K}_{j, h h}}\left\|\Delta_{k} f\right\|_{L_{T}^{\frac{2}{1-\alpha}} L^{p}}\left\|\Delta_{k^{\prime}} g\right\|_{L_{T}^{\frac{2}{1+\alpha}} L^{p}}
\end{aligned}
$$

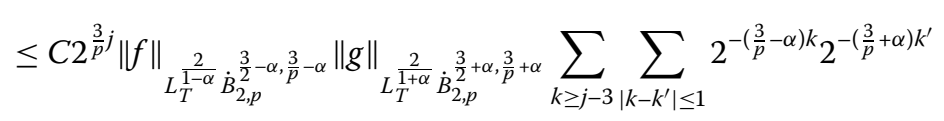

$$
\begin{aligned}
& \leq C 2^{-\frac{3}{p} j}\|f\|_{L_{T}^{1-\alpha} \dot{B}_{2, p}^{\frac{3}{2}-\alpha, \frac{3}{p}-\alpha}}\|g\|_{L_{T}^{1+\alpha} \dot{B}_{2, p}^{\frac{3}{2}+\alpha, \frac{3}{p}+\alpha}}
\end{aligned}
$$

and

$$
\begin{aligned}
\left\|I I I_{j, 2}+I I I_{j, 3}\right\|_{L_{T}^{1} L^{2}} & \leq C 2^{3 j\left(\frac{2}{p}-\frac{1}{2}\right)} \sum_{\left(k^{\prime}, k\right) \in \tilde{K}_{j, h h}}\left\|\Delta_{k} f \Delta_{k^{\prime}} g\right\|_{L_{T}^{1} L^{\frac{p}{2}}} \\
& \leq C 2^{-\frac{3}{2} j}\|f\|_{L_{T}^{1-\alpha} \dot{B}_{2, p}^{\frac{3}{2}-\alpha, \frac{3}{p}-\alpha}\|g\|_{L_{T}^{1+\alpha} \dot{B}_{2, p}^{2}} \frac{3}{2+\alpha, \frac{3}{p}+\alpha} .} .
\end{aligned}
$$

Summing up the estimates of $I I I_{j, 1} \sim I I I_{j, 4}$, we arrive at

$$
\begin{aligned}
& \sup _{2^{j}>\max \{|| \Omega \mid, N\}} 2^{\frac{3}{p} j}\left\|I I I_{j}\right\|_{L_{T}^{1} L^{p}}+\sup _{2^{j} \leq \max \{|\Omega|, N\}} 2^{\frac{3}{2} j}\left\|I I I_{j}\right\|_{L_{T}^{1} L^{2}} \\
& \quad \leq C\|f\|_{L_{T}^{1-\alpha} \dot{B}_{2, p}^{2}-\alpha, \frac{3}{p}-\alpha}\|g\|_{L_{T}^{1+\alpha}} \frac{2}{\dot{B}_{2, p}^{2}+\alpha, \frac{3}{p}+\alpha} .
\end{aligned}
$$

Then, combining (3.8)-(3.10) yields (3.7).

\section{The proof of Theorem 1.2}

The proof of Theorem 1.2 follows from the following standard Banach fixed point lemma combined with applications of the estimates established in the previous section.

Lemma 4.1 (Cannone [9]) Let $\left(\mathcal{X},\|\cdot\|_{\mathcal{X}}\right)$ be a Banach space, and $B: \mathcal{X} \times \mathcal{X} \rightarrow \mathcal{X} a$ bounded bilinear form satisfying $\left\|B\left(x_{1}, x_{2}\right)\right\|_{\mathcal{X}} \leq \eta\left\|x_{1}\right\|_{\mathcal{X}}\left\|_{x_{2}}\right\|_{\mathcal{X}}$ for all $x_{1}, x_{2} \in \mathcal{X}$ and some constant $\eta>0$. Then, if $0<\varepsilon<\frac{1}{4 \eta}$ and if $y \in \mathcal{X}$ such that $\|y\|_{\mathcal{X}} \leq \varepsilon$, then the equation $x=y+B(x, x)$ has a solution in $\mathcal{X}$ such that $\|x\|_{\mathcal{X}} \leq 2 \varepsilon$. This solution is the only one in the ball $\bar{B}(0,2 \varepsilon)$. Moreover, the solution depends continuously on $y$ in the following sense: if $\|\tilde{y}\|_{\mathcal{X}} \leq \varepsilon, \tilde{x}=\tilde{y}+B(\tilde{x}, \tilde{x})$ and $\|\tilde{x}\|_{\mathcal{X}} \leq 2 \varepsilon$, then

$$
\|x-\tilde{x}\|_{\mathcal{X}} \leq \frac{1}{1-4 \eta \varepsilon}\|y-\tilde{y}\|_{\mathcal{X}}
$$

Let $R_{j}(j=1,2,3)$ be the Riesz transforms on $\mathbb{R}^{3}$ and set $\widetilde{\mathbb{P}}=\left(\widetilde{\mathbb{P}}_{i j}\right)_{4 \times 4}$ with

$$
\widetilde{\mathbb{P}}_{i j}:= \begin{cases}\delta_{i j}+R_{i} R_{j}, & 1 \leq i, j \leq 3 \\ \delta_{i j} & \text { otherwise }\end{cases}
$$

where $\delta_{i j}$ is the Kronecker's delta notation. By using the Duhamel principle we easily obtain that problem (2.1) is equivalent to the following integral equation:

$$
v(t)=T_{\Omega, N}(t) v_{0}-B(v, v)(t)
$$


where

$$
B(v, v)(t):=\int_{0}^{t} T_{\Omega, N}(t-\tau) \widetilde{\mathbb{P}} \widetilde{\nabla} \cdot[v(\tau) \otimes v(\tau)] d \tau .
$$

Proof of Theorem 1.2 Let $\alpha \in\left(\frac{3}{2}-\frac{3}{p}, 1\right]$ be given and fixed, and let $X^{\alpha}$ be a Banach space endowed with the norm

$$
\|v\|_{X^{\alpha}}:=\|v\|_{\tilde{L} \frac{2}{1-\alpha}\left(0, \infty ; \dot{B}_{2, p}^{\frac{3}{2}-\alpha, \frac{3}{p}-\alpha}\right)}+\|v\|_{\tilde{L} \frac{2}{1+\alpha}\left(0, \infty ; \dot{B}_{2, p}^{\frac{3}{2}+\alpha, \frac{3}{p}+\alpha}\right)}
$$

Applying Lemma 3.1 with $\sigma=\frac{1}{2}$ and $\beta=-1+\frac{3}{p}$ leads to

$$
\left\|T_{\Omega, N}(t) v_{0}\right\|_{X^{\alpha}} \leq C_{0}\left\|v_{0}\right\|_{\dot{B}_{2, p}^{\frac{1}{2},-1+\frac{3}{p}}}
$$

for some constant $C_{0}>0$ and $v_{0} \in \dot{B}_{2, p}^{\frac{1}{2},-1+\frac{3}{p}}\left(\mathbb{R}^{3}\right)$.

Lemma 3.2 with $\sigma=\frac{1}{2}$ and $\beta=-1+\frac{3}{p}$ gives, for $v, w \in X^{\alpha}$ and some constant $C_{1}>0$,

$$
\begin{aligned}
\|B(v, w)\|_{X^{\alpha}} & =\left\|\int_{0}^{t} T_{\Omega, N}(t-\tau) \widetilde{\mathbb{P}} \widetilde{\nabla} \cdot[v(\tau) \otimes w(\tau)] d \tau\right\|_{X^{\alpha}} \\
& \leq C_{1}\|\widetilde{\nabla} \cdot[v(\tau) \otimes w(\tau)]\|_{\tilde{L}^{1}\left(0, \infty ; \dot{B}_{2, p}^{\frac{1}{2},-1+\frac{3}{p}}\right)} \\
& \leq C_{1}\|v\|_{X^{\alpha}}\|w\|_{X^{\alpha}},
\end{aligned}
$$

where we have used Lemma 3.3 for getting the last inequality.

Then, by Lemma 4.1, for any given $v_{0} \in \dot{B}_{2, p}^{\frac{1}{2},-1+\frac{3}{p}}\left(\mathbb{R}^{3}\right)$ satisfying

$$
\left\|v_{0}\right\|_{\dot{B}_{2, p}^{\frac{1}{2},-1+\frac{3}{p}}} \leq \frac{\epsilon}{C_{0}} \quad \text { with } 0<\epsilon<\frac{1}{4 C_{1}},
$$

we immediately see that there exists a unique solution $v$ of equation (4.1) in the ball with center 0 and radius $2 \epsilon$ in the space $X^{\alpha}$. Moreover, applying Lemmas 3.1-3.3 with $\alpha=1$, $\sigma=\frac{1}{2}$, and $\beta=-1+\frac{3}{p}$ implies that

$$
\begin{aligned}
\|v\|_{\tilde{L}^{\infty}\left(0, \infty ; \dot{B}_{2, p}\right.} \dot{\frac{1}{2}}^{\left.2,-1+\frac{3}{p}\right)} & \leq C\left\|v_{0}\right\|_{\dot{B}_{2, p}^{\frac{1}{2},-1+\frac{3}{p}}}+C\|v \otimes v\|_{\tilde{L}^{1}\left(0, \infty ; \dot{B}_{2, p}^{2}, \frac{3}{p}\right)} \\
& \leq C\left\|v_{0}\right\|_{\dot{B}_{2, p}^{\frac{1}{2},-1+\frac{3}{p}}}+C\|v\|_{X^{\alpha}}^{2}<\infty,
\end{aligned}
$$

which ensures $v \in\left[\tilde{L}^{\infty}\left(0, \infty ; \dot{B}_{2, p}^{\frac{1}{2},-1+\frac{3}{p}}\left(\mathbb{R}^{3}\right)\right)\right]^{4}$. Moreover, by using a standard density argument we can further infer that $v \in\left[C\left([0, \infty), \dot{B}_{2, p}^{\frac{1}{2},-1+\frac{3}{p}}\left(\mathbb{R}^{3}\right)\right)\right]^{4}$. This proves the global wellposedness assertion in Theorem 1.2.

The authors declare that they have no competing interests. 


\section{Acknowledgements}

The authors would like to thank Professor Shangbin Cui for helpful discussions. Project supported by the National Natural Science Foundation of China under the grant number 11571381.

Received: 11 November 2015 Accepted: 8 January 2016 Published online: 22 January 2016

\section{References}

1. Cushman-Roisin, B: Introduction to Geophysical Fluid Dynamics. Prentice Hall, Englewood Cliffs, New Jersey (1994)

2. Majda, A: Introduction to PDEs and Waves for the Atmosphere and Ocean. Courant Lecture Notes in Mathematics, vol. 9. New York University, Courant Institute of Mathematical Sciences, New York (2003)

3. Pedlosky, J: Geophysical Fluid Dynamics, 2nd edn. Springer, New York (1987)

4. Babin, A, Mahalov, A, Nicolaenko, B: On the regularity of three-dimensional rotating Euler-Boussinesq equations. Math. Models Methods Appl. Sci. 9, 1089-1121 (1999)

5. Fujita, H, Kato, T: On the Navier-Stokes initial value problem I. Arch. Ration. Mech. Anal. 16, 269-315 (1964)

6. Kato, $T$ : Strong $L^{p}$-solutions of the Navier-Stokes equation in $\mathbb{R}^{m}$, with applications to weak solutions. Math. Z. 187, 471-480 (1984)

7. Cannone, M: A generalization of a theorem by Kato on Navier-Stokes equations. Rev. Mat. Iberoam. 13, 515-541 (1997)

8. Koch, H, Tataru, D. Well-posedness for the Navier-Stokes equations. Adv Math 157, 22-35 (2001)

9. Cannone, M: Harmonic analysis tools for solving the incompressible Navier-Stokes equations. In: Friedlander, S, Serre, D (eds.) Handbook of Mathematical Fluid Dynamics, vol. III, pp. 161-244. Elsevier, Amsterdam (2004)

10. Lemarié-Rieusset, PG: Recent Developments in the Navier-Stokes Problems. Research Notes in Mathematics. Chapman \& Hall/CRC, Boca Raton (2002)

11. Chemin, J-Y, Desjardin, B, Gallagher, I, Grenier, E: Anisotropy and dispersion in rotating fluids. In: Nonlinear PDEs and Applications. Stud. Math. Appl., vol. 31. North-Holland, Amsterdam (2002)

12. Chemin, J-Y, Desjardins, B, Gallagher, I, Grenier, E: Mathematical Geophysics. An Introduction to Rotating Fluids and the Navier-Stokes Equations. Oxford Lecture Series in Mathematics and Its Applications, vol. 32. Clarendon, Oxford (2006)

13. Chen, Q, Miao, C, Zhang, Z: Global well-posedness for the 3 D rotating Navier-Stokes equations with highly oscillating initial data. Pac. J. Math. 262, 263-283 (2013)

14. Fang, D, Han, B, Hieber, M: Local and global existence results for the Navier-Stokes equations in the rotational framework. Commun. Pure Appl. Anal. 14, 609-622 (2015)

15. Giga, Y, Inui, K, Mahalov, A, Saal, J: Uniform global solvability of the rotating Navier-Stokes equations for nondecaying initial data. Indiana Univ. Math. J. 57, 2775-2791 (2008)

16. Hieber, M, Shibata, Y: The Fujita-Kato approach to the Navier-Stokes equations in the rotational framework. Math. Z. $265,481-491(2010)$

17. Iwabuchi, T, Takada, R: Global solutions for the Navier-Stokes equations in the rotational framework. Math. Ann. 357, 727-741 (2013)

18. Iwabuchi, T, Takada, R: Global well-posedness and ill-posedness for the Navier-Stokes equations with the Coriolis force in function spaces of Besov type. J. Funct. Anal. 267, 1321-1337 (2014)

19. Koh, Y, Lee, S, Takada, R: Dispersive estimates for the Navier-Stokes equations in the rotational framework. Adv. Differ. Equ. 19, 857-878 (2014)

20. Konieczny, P, Yoneda, T: On dispersive effect of the Coriolis force for the stationary Navier-Stokes equations. J. Differ. Equ. 250, 3859-3873 (2011)

21. Sun, J, Yang, M, Cui, S: Existence and analyticity of mild solutions for the 3D rotating Navier-Stokes equations. (submitted)

22. Charve, F: Global well-posedness and asymptotics for a geophysical fluid system. Commun. Partial Differ. Equ. 29, 1919-1940 (2004)

23. Charve, F: Global well-posedness for the primitive equations with less regular initial data. Ann. Fac. Sci. Toulouse 17, $221-238(2008)$

24. Charve, F, Ngo, V: Global existence for the primitive equations with small anisotropic viscosity. Rev. Mat. Iberoam. 27, 1-38 (2011)

25. Koba, H, Mahalov, A, Yoneda, T: Global well-posedness for the rotating Navier-Stokes-Boussinesq equations with stratification effects. Adv. Math. Sci. Appl. 22,61-90 (2012)

26. Babin, A, Mahalov, A, Nicolaenko, B: On the asymptotic regimes and the strongly stratified limit of rotating Boussinesq equations. Theor. Comput. Fluid Dyn. 9, 223-251 (1997)

27. Babin, A, Mahalov, A, Nicolaenko, B: Fast singular oscillating limits and global regularity for the 3D primitive equations of geophysics. Special issue for R. Temam's 60th birthday. Math. Model. Numer. Anal. 34, 201-222 (2000)

28. Babin, A, Mahalov, A, Nicolaenko, B: Strongly stratified limit of 3D primitive equations in an infinite layer. In: Advances in Wave Interaction and Turbulence. Contemp. Math., vol. 283 (2001)

29. Charve, F: Convergence of weak solutions for the primitive system of the quasigeostrophic equations. Asymptot. Anal. 42, 173-209 (2005)

30. Charve, F: Asymptotics and lower bound for the lifespan of solutions to the Primitive Equations. arXiv:1411.6859

31. Chemin, J-Y: A propos d'un problème de pénalisation de type antisymétrique. J. Math. Pures Appl. 76, 739-755 (1997) (in French)

32. Danchin, R: Global existence in critical spaces for compressible Navier-Stokes equations. Invent. Math. 141, 579-614 (2000)

33. Danchin, R: Global existence in critical spaces for flows of compressible viscous and heat-conductive gases. Arch. Ration. Mech. Anal. 160, 1-39 (2001)

34. Bahouri, H, Chemin, J-Y, Danchin, R: Fourier Analysis and Nonlinear Partial Differential Equations. Springer, Berlin (2011)

35. Triebel, H: Theory of Function Spaces. Monograph in Mathematics, vol. 78. Birkhäuser, Basel (1983)

36. Bony, J-M: Symbolic calculus and propagation of singularities for nonlinear partial differential equations. Ann. Sci. Éc. Norm. Super. 14, 209-246 (1981) (in French) 